\title{
Performance of Magnetorheological Fluids Flowing Through Metal Foams
}

\author{
X.h Liu ${ }^{1}$, Z.m Fu${ }^{1}$, X.y $\mathrm{Yao}^{2}, \mathrm{~F} \mathrm{Li}^{1}$ \\ ${ }^{1}$ School of Mechanical Engineering, Shanghai institute of Technology, 201418, Shanghai, China \\ ${ }^{2}$ College of Optoelectronic Engineering, Chongqing University, 400044, Chongqing, China, liu_xuhui79@163.com
}

\begin{abstract}
If magnetorheological (MR) fluids are stored in porous materials, when excited by the external magnetic field, MR fluid will be drawn out and produce MR effect, which could be used to solve the following problems of the MR damper, such as the seal, volume and the cost of MR fluid damper. In this paper, the effect of structure of metal foams on the performance of MR fluid is investigated; the relationship between the penetrability and the porosity of the metal foams is measured, the change of MR fluid performance flowing though the metal foams is obtained. It shows that, after flowing through metal foams, the change of performance of MR fluid is about $2.5 \%$. Compared to the sponge, the porous metal foams have the obvious advantages in high porosity and rigidity, which provide a convenient and low-cost way to design the MR damper.
\end{abstract}

Keywords: Metal foams, penetrability, porosity, MR fluid

\section{INTRODUCTION}

$\mathrm{P}$ OROUS MATERIALS, such as the sponge, sintered metal, felt or fabric, used to design and solve the following problems, such as the seal, required volume and cost of the MR damper, are studied widely [1-3]. Carlson [1] developed the controllable MR sponge dampers, which are also used to control the vibration of washing machines. Their investigations show that, in a direct shear mode, the novel design can produce the force of $100 \mathrm{~N}$, but only $3 \mathrm{ml}$ MR fluid is needed, that is, a lower volume of MR fluid in the shear gap than the usual design. Besides, the design is without the seals, bearings or precision mechanical tolerances.

The structures of metal foams, such as the porosity, the penetrability, are the key parameters that decide the achievable damping forces. G. Bossis [4] investigated the flowing model and pressure drop of MR fluid through the porous media theoretically; the calculation model is also built to describe the flowing process. V. Bashtovoi [5] studied the phenomena of capillary rise of MR fluid and built the model of surface distortion; however, the performance of MR fluid after flowing through metal foams is seldom investigated.

Metal foam has a very high porosity, that is, nearly $75-95 \%$ of the volume of the material is the open pore, besides, it possesses bigger stiffness than the usual polyurethane sponge, and it has often served as a new functional material for various purposes, such as the catalyst carrier, the filters, the absorbers, and so on. Metal foam is mainly made of metal, usually, nickel $(\mathrm{Ni})$, copper $(\mathrm{Cu})$, ferrum $(\mathrm{Fe})$ and aluminum $(\mathrm{Al})$. Harte [6] has investigated the fatigue strength of sandwich beam with aluminum alloy foam; J. Banhart [7] has also developed metal foams and tested their structure; from their investigations, the main advantages of these foams are the high porosity and stability with low weight, as mentioned above.

As shown in reference [1] and [3], Carlson presented the new MR sponge dampers; however, there are many shortcomings for the design using the sponge, such as its stiffness and durability, which will shorten the life of dampers. Thus, it is very important for the design to test the open porosity and rigidity. Here, metal foams, used to replace the sponge, will improve the MR sponge fluid, and provide a wide research region for MR damper using porous materials. Our previous investigations [8][9] show that MR fluid stored in metal foams will produce the MR effect once excited, which also shows the feasibility of replacement experimentally. However, the effect of structure of metal foams on the performance of MR fluid has been seldom investigated. In fact, when MR fluid flows through the metal foams, the influence of the structure parameter on the performance of MR fluid is the most important factor.

In this paper, according to the particle size of MR fluid produced by Lord.co (USA), a series of metal foams with high porosity and rigidity will be investigated, besides, the method of testing the structure parameter of metal foams is also provided, accordingly, the relationship between the change of MR fluid and the structure parameter of metal foams is also studied.

\section{MATERIALS AND PARAMETERS OF MEASUREMENT}

Based on the design of MR damper, the metal foams will satisfy: 1. the pore size of metal foams must be appropriate, if the size is too big, the capillary effect will be unobvious and MR fluid will leak from the damper; if too small, most of magnetic particles will be blocked, and unable to flow through the metal foams, therefore, the performance of MR fluid will be greatly influenced. 2 . In order to insure the stored volume of MR fluid in metal foams, the high porosity should be suitable to store MR fluid; 3. Compared to sponge, the bigger rigidity will be necessary to resist wear and distortion.

MRF-132AD produced by Lord.co is selected as the test specimen for the experiment; the particle size and the shape distributions of the magnetizable particles were examined by scanning electron microscopy (SEM), as shown in Fig.1. The MR particle shape was found to be nearly spherical and 
typical dimensions were around 1-5 $\mu \mathrm{m}$ in diameter. The carrier liquid is hydrocarbon based oil of around 30\% iron particles, the other characteristic of which is as [10];

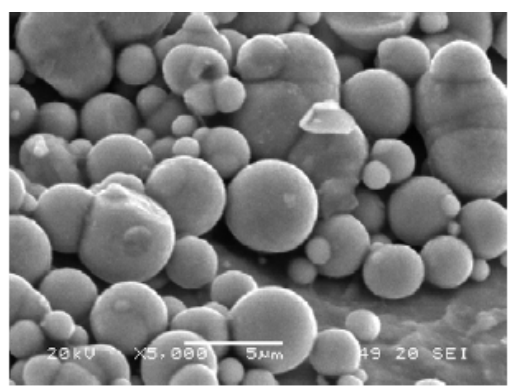

Fig.1. The particle of MR fluid.

The production process of metal foams is shown in Fig.2. The pore size and thickness of the polyurethane (model) can be adjusted on the basis of the experimental demand, such as the open porosity is from 50 ppi to 140 ppi (pores per inch), and the thickness is about $2.0 \mathrm{~mm}$. In order to make the non-conducting foam polyurethane conductive and electrodeposit the metal, the polyurethane should be pretreated, the materials used to pretreat are industrial reagent and special conducting paint; the process is mainly as follows:

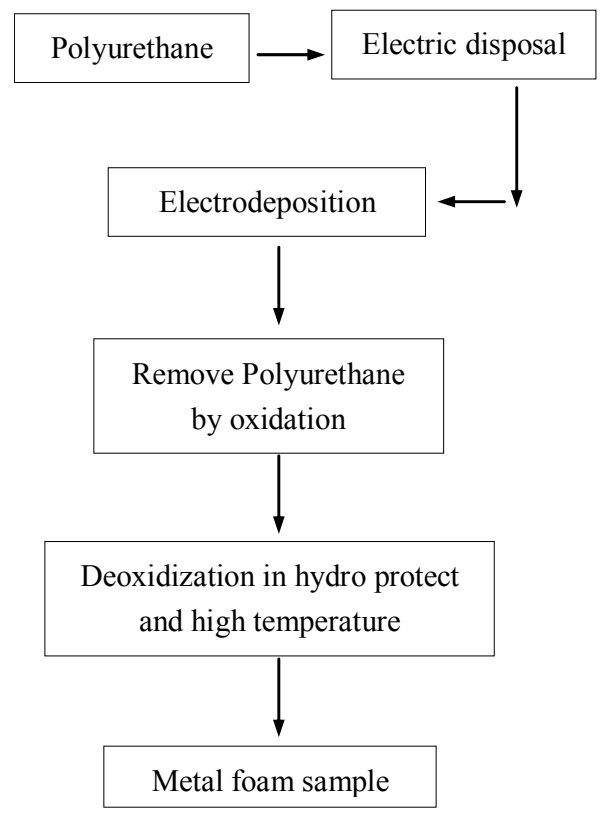

Fig.2. T he production process of metal foams.

\section{Roughening and deoxidization}

In order to enhance the porosity and wettability, the model (that is, the polyurethane) will be soaked in acidic solution with potassium permanganate and be roughened first, and then deoxidized in oxalic acid solution.

\section{Preparation of conducting layer}

The special conductive adhesive (namely, carbon-containing dope) will be spread on the surface of pretreated polyurethane, then dried, thus, the non-conducting foam polyurethane will be made conductive; the polyurethane will be soaked in the salt solution (a certain metal element, such as copper, nickel, etc. dissolved in the solution), so that the polyurethane could be electrodeposited on the metal element.

\section{Thermal treatment}

That is, the deoxidization of the metal foams and riddance of the polyurethane and deoxidization of the metal oxide, thus, the mechanical property of the metal foams will be greatly improved.

The metal foams with various porosity and rigidity are obtained by changing the current density and mold (or polyurethane); the structure of metal foams is shown in Fig.3.

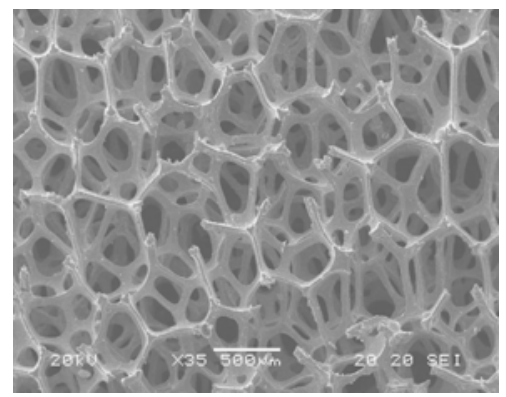

Fig.3. The metal foams (SEM).

According to the production process mentioned above, the metal foams can be considered as open-cell metals; from Fig. 3, the pore size of the metal foams is from $100 \mu \mathrm{m}$ to $550 \mu \mathrm{m}$, which is larger than the particle size of MR fluid (which is about $3.3 \mu \mathrm{m}$ ).

\section{METHODS OF MEASUREMENT}

As MR fluid is considered to be the suspending liquid with the solid and liquid phases, in order to obtain the change of performance of MR fluid after flowing through the metal foams, the following assumptions are made,

- MR fluid is considered as the incompressible fluid;

- The flowing state is laminar flow;

- The temperature is invariable;

Navier-Stokes equation on the fluid given [11]:

$$
\rho \frac{d v}{d t}=-\nabla p+\rho F+\mu \Delta v
$$

Here, $\rho$ is the density of fluid; $v$ is the velocity vector, if in the rectangular coordinate system, the component is $u, v$, $w ; p$ is the isotropy pressure; $F$ is the body force, if in the rectangular coordinate system, the component is $X, Y, Z ; \mu$ is the dynamic viscosity coefficient; thus, the equation (1) is transformed:

$$
\left.\begin{array}{r}
\rho \frac{d u}{d t}=-\frac{\partial p}{\partial x}+\rho X+\mu \Delta u \\
\rho \frac{d v}{d t}=-\frac{\partial p}{\partial y}+\rho Y+\mu \Delta v \\
\rho \frac{d w}{d t}=-\frac{\partial p}{\partial z}+\rho Z+\mu \Delta w
\end{array}\right\}
$$


According to [12] and formulas (1) and (2), when the MR fluid flows through the metal foams,

$$
\frac{Q}{A}=B \frac{\Delta p}{\eta \delta}
$$

Here, $Q$ is the flux of MR fluid, $A$ is the cross-section of channels, $B$ is the permeability, $\Delta p$ is the pressure difference, $\eta$ is the viscosity, $\delta$ is the thickness of metal foams.

Under the circumstances of steady flowing,

$$
B=\frac{Q}{A} \frac{\eta \delta}{\Delta p}
$$

As a rule, the relative permeability $K$ is often expressed as,

$$
K=\frac{B}{\eta \delta}
$$

The permeability of the metal foams is considered as that, in a given pressure, the performance of the fluid flows through the porous materials; it rests with the characteristics of the fluid, the porosity and the pore shape of the materials and the thickness.

The porosity $\theta$ can be described as:

$$
\theta=\frac{\text { the total volume of pores } \times 100}{\text { the total volume of the porous metallic foams }} \%
$$

The melted paraffin is adopted to test the porosity $\theta$, the method is as follows:

1. The regular metal foams are prepared, and the mass $m_{l}$ and the volume $V_{l}$ is obtained, here, the volume $V_{l}$ is the total volume of the metal foams;

2 . The density $\rho$ of the paraffin is obtained by measuring cylinder; the main principle adopted is buoyancy law;

3. Paraffin is melted, the metal foams are put into and soaked in the paraffin; then the melted state is kept for about ten minutes till the metal foams are fully immerged in the melted paraffin, then the foam is cooled;

4. Scrape off the paraffin on the surface of the metal foams, the mass $m_{2}$ is obtained; the difference value $m_{2}-m_{l}$ is the mass of the paraffin soaked into the metal foams. Then,

$$
\theta=\frac{m_{2}-m_{1} / \rho}{V_{1}} \times 100 \%
$$

The porosity of five selected metal foams is summarized in Table 1.

Table 1 . The porosity of the metal foams

\begin{tabular}{cccccc}
\hline$\%$ & $\mathrm{a}^{\#}$ & $\mathrm{~b}^{\#}$ & $\mathrm{c}^{\#}$ & $\mathrm{~d}^{\#}$ & $\mathrm{e}^{\#}$ \\
\hline$\theta$ & 65.51 & 59.41 & 82.05 & 75.27 & 68.14 \\
\hline
\end{tabular}

\section{RESULTS AND DISCUSSION}

The experimental setup is shown in Fig.4.

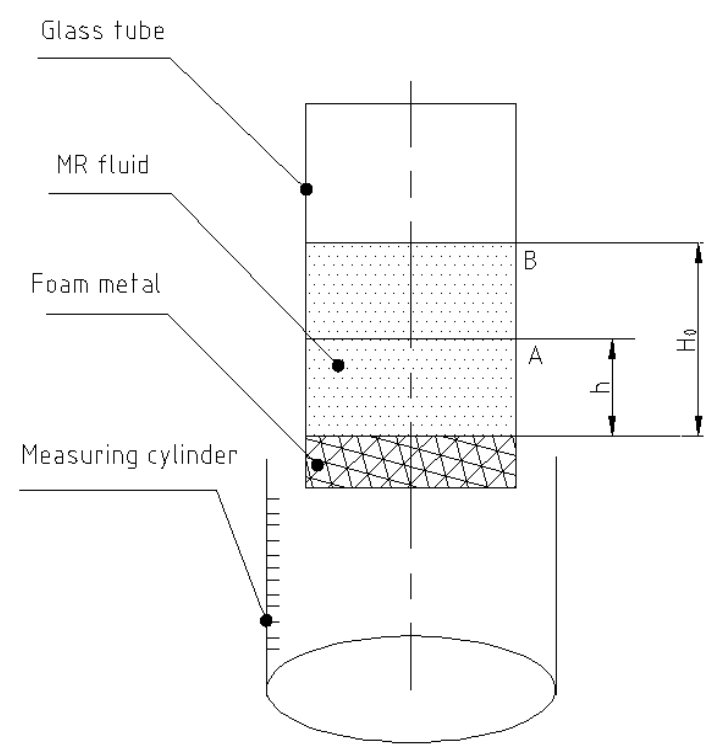

Fig.4. Schematic view of the experimental test rig.

The test rig consists of the metal foams, the glass tube and measuring cylinder (the measuring cylinder is put on the electronic balance first), the density of MR fluid is measured by measuring cylinder and the indication of electronic balance, here, the diameter of measuring cylinder is measured by calipers; the metal foam is adhered to the bottom of the glass tube.

Measurements are carried out at room temperature, when the volume of MR fluid in the measuring cylinder is $\mathrm{N} \mathrm{ml}$, this is the original time $t=t_{0}=0$, here, the parameter $\mathrm{N}$ can be chosen by the experiments, the height of liquid (here is MR fluid) in glass tube is $H_{0}$, corresponding to the original time, the pressure put on the metal foam is $\rho_{0} \mathrm{~g} H_{0}$, and $\rho_{0}$ is the original density of MR fluid. When the time is $t$, the height of the liquid changes from $H_{0}$ to $H(t)$, that is, the total volume flowing through the metal foams from $t_{0}$ to $t$ is $S_{0}\left[H_{0}\right.$ $-H(t)]$, therefore, the formulas (4) and (5) can be transformed:

$$
S_{0}\left[H_{0}-H(t)\right]=K A \int_{t_{0}}^{t} \rho_{0} g H(t) d t
$$

Where $S_{0}$ is the cross-section of the glass tube and is equal to $A, K$ is the relative penetrability, $\mathrm{m}^{2} \cdot \mathrm{s} / \mathrm{kg} ; t_{0}$ is equal to zero,

$$
H_{0}-H(t)=K \int_{0}^{t} \rho_{0} g H(t) d t
$$

That is

$$
K t=\frac{\ln H_{0}-\ln h}{\rho_{0} g}
$$


Here, $h$ is the height in the time $t$, if the height of liquid in the time $t$ is measured, then the relative penetrability $K$ will be obtained.

According to the inner diameters of the measuring cylinder, the difference of the volume increased in the measuring cylinder can be changed into the difference of the height in the glass tube; the following formula (10) is obtained

$$
K=\frac{\ln H_{0}-\ln h}{\rho_{0} g t}=\frac{\ln H_{0}-\ln \left(H_{0}-\frac{4 V}{\pi \phi^{2}}\right)}{\rho_{0} g t}
$$

The penetrability is compared to Taylor experimental formula [11], as shown in Fig.4.

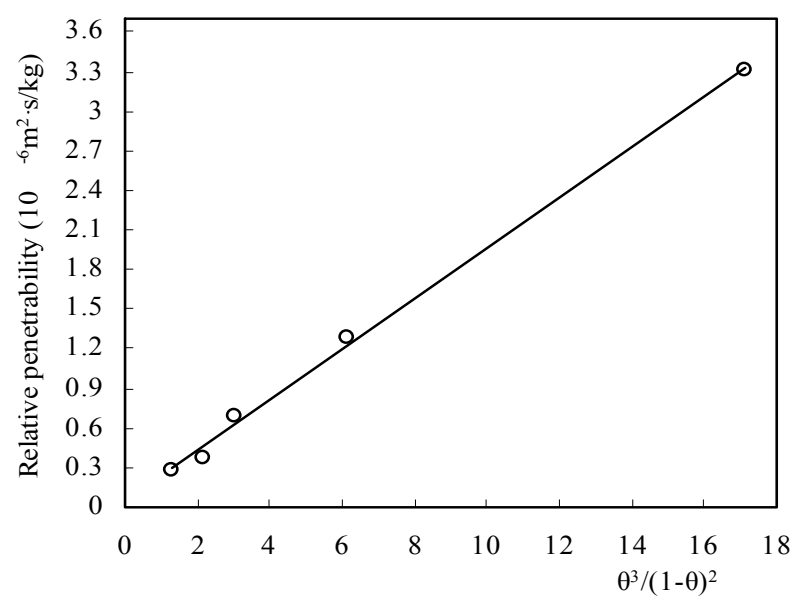

Fig.5. Relative penetrability vs. the porosity of metal foams. Here, the symbols (circles) show the experimental data, the solid line is a simulation with the experiential formula [12][13][14].

The initial density of MR fluid is $\rho_{0}=2.71 \mathrm{~g} / \mathrm{cm}^{3}$; the collected density is shown in Fig.6.

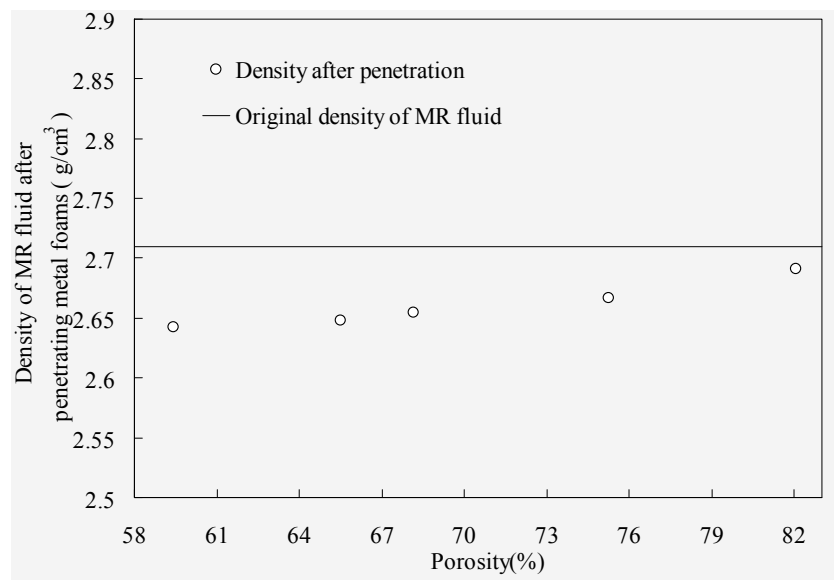

Fig.6. Density of MR fluid after flowing through the metal foams vs. the porosity. The symbols (circles) show the experimental data for the density of MR fluid through the porous metal with different porosity, the solid line shows the original density of MR fluid.
Fig.6 also shows that with the porosity of metal foam increased, the density of MR fluid flowing through the metal foams will increase.

After MR fluid flowing through the metal foams, the maximal error bands are made by the $b^{\#}$ metal foams:

$$
\Delta=\frac{\rho_{0}-\rho}{\rho_{0}} \times 100 \%=\frac{2.71-2.642}{2.71} \times 100 \%=2.5 \%
$$

Here, the performance of MR fluid flowing through the metal foams is considered as nearly invariable (the maximal error is $2.5 \%$ ), which is mostly due to the pore size of the metal foams that is larger than the particle size of MR fluid, that is, after flowing through the metal foams, the loss (mainly resulting from clogging) of iron particles could be negligible.

However, as the color of MR fluid is nearly black, it is difficult to obtain the change of the height in glass tube by visual observation or optical instruments; in this work, the measuring cylinder is adopted to test the liquid drop, due to the continuity of MR fluid in different pressure, the volume of the partial liquid flowing through the metal foams, but not arriving to the measuring cylinder is estimated, which will bring out the difference.

\section{CONCLUSION}

The paper presents the influence of structure parameters of the metal foams on MR fluid; the results show that the performance of MR fluid changes little after flowing through metal foams. As the foam metals have the obvious advantages in high rigidity, compared to the sponge, they can be used to design the novel and low-cost MR damper.

\section{ACKNOWLEDGMENTS}

The work described in this paper was fully supported by the National Natural Science Foundation of China (No. 51105256).

\section{REFERENCES}

[1] Carlson, J.D. (2001). Sponge wrings cost from MR fluid devices. Machine Design, 22 (2), 73-76.

[2] Guldbakke, J.M., Hesselbach, J. (2006). Development of bearings and a damper based on magnetically controllable fluids. Journal of Physics: Condensed Matter, 18, S2959-S2972.

[3] Carlson, J.D., Jolly, M.R. (2000). MR fluid, foam and elastomer devices. Mechatronics, 10, 555-569.

[4] Kuzhir, P., Bossis, G. (2003). Flow of MR fluid through porous media. European Journal of Mechanics, 22, 331-343.

[5] Bashtovoia, V., Bossis, G. (2005). Magnetic field effect on capillary rise of magnetic fluids. Journal of Magnetism and Magnetic Materials, 289, 376-378.

[6] Harte, A.M., Fleck, N.A., Ashby, M.F. (2001). The fatigue strength of sandwich beam with an aluminum alloy foam core. International Journal of Fatigue, 23, 499-507. 
[7] Banhart, J. (2001). Manufacture, characterization and application of cellular metals and metal foams. Progress in Material Science, 46, 6-17.

[8] Liu, X.H., Wong, P.L., Wang, W., Bullough, W.A. (2010). Feasibility study on storage of MR Fluid using metal foams. Journal of Intelligent Material Systems and Structures, 21, 1193-1200.

[9] Liu, X.H. (2010). Shear performance of novel disk-type porous foam metal magneto-rheological (MR) fluid actuator. Optoelectronics and Advanced Materials - Rapid Communications, 9, 1346-1349.

[10] LORD Corporation. Hydrocarbon-Based MR Fluid $M R F-132 A D$. Retrieved from www.lord.com.
[11] Taylor, D.W. (1948). Fundamentals of Soil Mechanics. New York: John Wiley \& Sons.

[12] Herrmann, H.J., Andrade Jr., J.S., Araújo, A.D. (2007). Particles in fluids. Computer Physics Communications, 177, 158-161.

[13] Belforte, G., Raparelli, T., Viktorov, V., Trivella, A. (2007). Permeability and inertial coefficients of porous media for air bearing feeding systems. Journal of Tribology, 129, 705-711.

[14] Chen, Y., Shi, M. (2000). Determination of permeability for porous media using fractal theory[J]. Journal of Tsinghua University, 40, 94-99.

Received July 4, 2011. Accepted October 21, 2011. 